Japan. J. Math.

Vol. 28, No. 2, 2002

\title{
Hopf-equivalences for zero-dimensional dynamical systems
}

\author{
By Hisatoshi Yuasa \\ (Received June 10, 2002) \\ (from Tokyo Journal of Mathematics)
}

\begin{abstract}
In the class of zero-dimensional dynamical systems, we see some links among Hopf-equivalences, coboundaries, hitting times and recurrence properties for dynamical systems. Each of the first three notions concerns with a relation between an arbitrary pair of nonempty clopen sets.
\end{abstract}

\section{Introduction}

In [8], for a non-singular and bi-measurable transformation $T$ on a Lebesgue space $(\Omega, \mathcal{B}, \mu)$, E. Hopf introduced the following relation between any pair of measurable sets $A, B \in \mathcal{B}$ with strictly positive measures: there exist a sequence $\left\{n_{i} ; i \geq 1\right\}$ of integers, and two sequences $\left\{A_{i} ; i \geq 1\right\}$ and $\left\{B_{i} ; i \geq 1\right\}$ of pairwise disjoint measurable subsets of $A$ and $B$, respectively, such that

$$
\begin{gathered}
\mu\left(A \backslash \bigcup_{i=1}^{\infty} A_{i}\right)=0, \quad \mu\left(B \backslash \bigcup_{i=1}^{\infty} B_{i}\right)=0 \text { and } \\
B_{i}=T^{n_{i}} A_{i} \text { for every integer } i \geq 1 .
\end{gathered}
$$

This relation gives an equivalence relation, the so-called Hopf-equivalence. E. Hopf showed in [8] that a non-singular and bi-measurable transformation on a Lebesgue space has an equivalent finite invariant measure if and only if there exists no measurable subset of the space which is Hopf-equivalent under the transformation to its proper subset. Then, the Hopf-equivalence was found to play fundamental and crucial roles in analyses of measure-theoretical orbit structures of non-singular and bi-measurable transformations on Lebesgue spaces. And after that, it was discovered by T. Giordano, R. Herman, I. Putnam and C. Skau [3, 4, 7] and E. Glasner and B. Weiss [5] that some relations similar to the Hopf-equivalence played fundamental and crucial roles even in the analyses of topological orbit structures of

2000 Mathematics Subject Classification. Primary 37B99, Secondary 37B20.

Key words and Phrases. Hopf-equivalence, recurrence property, hitting times, zerodimensional dynamical system. 
minimal homeomorphisms on Cantor sets. One of such similar relations is stated as follows. For a minimal homeomorphism $\phi$ on a Cantor set $X$, and an arbitrary pair of nonempty clopen subsets $A$ and $B$ of $X$,

(a) there exist integers $n_{i}, 1 \leq i \leq k$, and two partitions $\left\{A_{i} ; 1 \leq i \leq k\right\}$ and $\left\{B_{i} ; 1 \leq i \leq k\right\}$ of $A$ and $B$ into (finitely many) clopen subsets, respectively, such that $B_{i}=\phi^{n_{i}}\left(A_{i}\right)$ for every integer $1 \leq i \leq k$.

It was a significant step in the analyses of the topological orbit structures to discover that Condition (a) is equivalent to the following cohomological condition for a dynamical system:

(b) The difference $\chi_{A}-\chi_{B}$ of the characteristic functions of $A$ and $B$ is a coboundary, i.e., there exists an integer-valued continuous function $f$ on $X$ such that $\chi_{A}-\chi_{B}=f \circ \phi-f$.

This equivalence was exhibited in [4, Lemma 3.3]. Notice that Condition (a) implies Condition (b) for any homeomorphism $\phi$ on a zero-dimensional compact Hausdorff space $X$. One of the main points of this paper is that we deal with a condition, which is different from both (a) and (b) above, but is closely related to them. This condition is also concerned with a relation between an arbitrary pair of nonempty clopen subsets and stated as follows:

(c) For any $x \in A \cup B$, there exists an integer $n \geq 1$ such that

$$
\sum_{j=0}^{n-1} \chi_{A}\left(\phi^{j} x\right)=\sum_{j=0}^{n-1} \chi_{B}\left(\phi^{j} x\right) .
$$

When T. Giordano, I. Putnam and C. Skau showed in [4, Lemma 3.3], by using a refining sequence of Kakutani-Rokhlin partitions, that Condition (b) implies Condition (a) for a minimal homeomorphism on a Cantor set, Condition (c) was used as an auxiliary condition for proving the implication. It may be worth noticing that Condition (c) is not an equivalence relation in general, although both (a) and (b) are.

Then, the following two questions occurred to the author. Under the hypothesis that $\phi$ is just a homeomorphism on a zero-dimensional compact Hausdorff space $X$,

(I) What relationships hold among Conditions (a), (b) and (c)?

(II) What are intrinsic properties of $\phi$ under which Conditions (a), (b) and (c) are all equivalent?

In Theorems 5.1 and 5.3, we will obtain two results concerning (I). Theorem 5.1 says that if $\phi$ is pointwise chain recurrent, i.e., any point in $X$ is chain recurrent for $\phi$, then Condition (c) implies Condition (a). Proposition 3.4, which is exploited in the proof of Theorem 5.1, gives a topological counterpart of an immediate consequence of $[8$, Theorem 5] that a non-singular and bi-measurable transformation on 
a Lebesgue space is recurrent if and only if no measurable set with strictly positive measure is finitely Hopf-equivalent under the transformation to its proper subset. When $\phi$ has no additional properties such as the pointwise chain recurrence, Condition (c) will turn out in Theorem 5.3 to be sufficient for the validity of another relation similar to the Hopf-equivalence:

(d) For nonempty clopen subsets $A$ and $B$ of $X$, there exist a sequence of integers $\left\{n_{i} ; i \geq 1\right\}$, and two partitions $\left\{A_{i} ; i \geq 1\right\}$ and $\left\{B_{i} ; i \geq 1\right\}$ of $A$ and $B$ into countable closed subsets, respectively, such that $B_{i}=\phi^{n_{i}} A_{i}$ for every integer $i \geq 1$.

As for (II), it will be shown also in Corollary 5.7 that, under the hypothesis that $\phi$ is just a homeomorphism on a zero-dimensional compact Hausdorff space $X$, Conditions (a), (b) and (c) are all equivalent if and only if any point in $X$ is positively recurrent for $\phi$. Although the last condition concerning a recurrence property is satisfied in the case when $\phi$ is a minimal homeomorphism on a Cantor set $X$, the condition is not necessarily satisfied by every homeomorphism for which Conditions (a) and (b) are equivalent. That is to say the equivalence of (a), (b) and (c) are strictly stronger than that of (a) and (b), which may be noted from the fact that Condition (c) is not necessarily an equivalence relation. As an example of such a class of homeomorphisms not satisfying the condition, we will introduce a class of almost minimal homeomorphisms on Cantor sets which were dealt with by A. Danilenko in [2].

In concluding this section, the author hopes that some readers will find it interesting to see that the proof of Corollary 5.7, without using any refining sequences of Kakutani-Rokhlin partitions, i.e., the Bratteli-Vershik representation [7], gives a proof of the fact that Condition (b) implies Condition (a) under the hypothesis that any point of $X$ is positively recurrent.

The author is grateful to Toshihiro Hamachi, Yuji Ito and Fumiaki Sugisaki for helpful comments, especially, some comments of Yuji Ito inspired the author to make Theorem 5.1 much better than the previous version.

\section{Hopf-equivalences}

Throughout this paper, we will restrict our attentions to the class of homeomorphisms on zero-dimensional compact Hausdorff spaces. The term "dimensional" means the covering dimension of a normal topological space; see [9, Definition 8.1]. The following proposition enables us to view any zero-dimensional compact Hausdorff space in a familiar fashion, which can be seen on page 47 of [9].

Proposition 2.1. A compact Hausdorff space $X$ has the covering dimension zero if and only if $X$ is homeomorphic to a nonempty and closed subset of the product space of finite discrete spaces. 
Let $\phi$ be a homeomorphism on a zero-dimensional compact Hausdorff space $X$.

Definition 2.2. A homeomorphism $\alpha$ from a nonempty clopen set $A \subset X$ to a nonempty clopen set $B \subset X$ is called a $\phi$-homeomorphism if there exists an integer-valued function $t: A \rightarrow \mathbb{Z}$, which is called a time change function of $\alpha$, such that

$$
\alpha(x)=\phi^{t(x)}(x) \text { for any } x \in A .
$$

For such a $\phi$-homeomorphism $\alpha$, we denote by $\operatorname{Dom}(\alpha)$ and $\operatorname{Im}(\alpha)$ the domain $A$ of $\alpha$ and the image $B$ of $\alpha$, respectively. We denote by $[\phi]_{*}$ the set of $\phi$-homeomorphisms and denote by $[[\phi]]_{*}$ the set of $\phi$-homeomorphisms with continuous time change functions.

The term " $\phi$-homeomorphism" is not commonly used, but is introduced here following the notion of " $T$-map" defined on page 61 of [6]. In general, there is an ambiguity for choosing a time change function of a $\phi$-homeomorphism, i.e., the values of a time change function at a point $x$ can vary if and only if $x$ is a periodic point for $\phi$.

Definition 2.3. Two nonempty clopen subsets $A$ and $B$ of $X$ are said to be finitely Hopf-equivalent if Condition (a) in the preceding section holds.

Two nonempty clopen sets $A$ and $B$ of $X$ are finitely Hopf-equivalent if and only if there exists a $\phi$-homeomorphism with a continuous time change function whose domain and image are $A$ and $B$, respectively.

Definition 2.4. Two nonempty clopen subsets $A$ and $B$ of $X$ are said to be countably Hopf-equivalent if Condition (d) in the preceding section holds.

If there exists a $\phi$-homeomorphism $\alpha$ such that $\operatorname{Dom}(\alpha)=A$ and $\operatorname{Im}(\alpha)=B$, then it is clear that $A$ and $B$ are countably Hopf-equivalent. However, in general, it is unknown whether the converse is true, or not.

Remark 2.5. In [1, Theorem 4.8], S. Bezugyli and J. Kwiatkowski gave some necessary and sufficient conditions for the class of minimal homeomorphisms on Cantor sets so that the finite and countable Hopf-equivalences are equivalent.

The following topological counterpart of [8, Theorem 5] can be proven in the same way as the proof of $[8$, Theorem 5$]$ :

Lemma 2.6. For a homeomorphism $\phi$ on a zero-dimensional compact Hausdorff space $X$, the following are equivalent:

(i) There exist a clopen subset $U$ of $X$ which is finitely Hopf-equivalent to a proper subset of $U$; 
(ii) There exists a clopen subset $U$ of $X$ such that $\phi(U)$ is a proper subset of $U$.

\section{Recurrence properties}

In this section, we shall recall some recurrence properties for topological dynamical systems. Let $\phi$ be a homeomorphism on a compact Hausdorff space $X$. A subset $S \subset \mathbb{Z}$ (or $\mathbb{Z}_{+}:=\{0,1,2, \ldots\}$ ) is said to be relatively dense in $\mathbb{Z}$ (or $\mathbb{Z}_{+}$) if there exists an integer $K>0$ such that $[n, n+K) \cap S \neq \emptyset$ for every $n \in \mathbb{Z}$ (or $\mathbb{Z}_{+}$).

Definition 3.1. (i) A point $x \in X$ is said to be positively recurrent for $\phi$ if for any open neighborhood $U$ of $x$ there exists a strictly positive integer $n$ such that $\phi^{n} x \in U$.

(ii) A point $x \in X$ is said to be recurrent for $\phi$ if it is positively recurrent for each of $\phi$ and $\phi^{-1}$.

(iii) A point $x \in X$ is said to be uniformly recurrent for $\phi$ if for any nonempty open neighborhood $U$ of $x$ the set $\left\{n \in \mathbb{Z} ; \phi^{n}(x) \in U\right\}$ is a relatively dense subset of $\mathbb{Z}$.

(iv) We say that $\phi$ is pointwise positively recurrent (respectively, pointwise recurrent) if any point in $X$ is positively recurrent (respectively, recurrent) for $\phi$.

(v) We say that $\phi$ is pointwise uniformly recurrent if any point in $X$ is uniformly recurrent for $\phi$.

The notion of uniform recurrence is often referred to as almost periodicity in several literatures. A pointwise recurrent homeomorphism on a compact Hausdorff space is pointwise uniformly recurrent. There always exist recurrent points because Zorn's lemma implies the existence of a minimal set for $\phi$.

Next, we shall introduce another recurrence property, the so-called chain recurrence, which is weaker than any of those defined above:

Definition 3.2. (i) Let $\mathcal{V}=\left(V_{i}\right)_{i \in I}$ be an open covering of $X$. A sequence $\omega=(\omega(n))_{n \in \mathbb{Z}}$ of elements in $I$ is called a $\mathcal{V}$-pseudo-orbit of $\phi$ if

$$
V_{\omega(n)} \cap \phi^{-1} V_{\omega(n+1)} \neq \emptyset \text { for every } n \in \mathbb{Z} .
$$

(ii) A point $x \in X$ is said to be chain recurrent for $\phi$ if for any open covering $\mathcal{V}=\left(V_{i}\right)_{i \in I}$ of $X$ and $i \in I$ with $x \in V_{i}$, there exists a periodic $\mathcal{V}$-pseudo-orbit $\omega=(\omega(n))_{n \in \mathbb{Z}}$ of $\phi$ such that $\omega(0)=i$.

(iii) We say that $\phi$ is pointwise chain recurrent if any point in $X$ is chain recurrent for $\phi$.

The notion of chain recurrence was introduced by C. C. Conley. The definitions (i) and (ii) in Definition 3.2 are given following [10] where M. Pimsner referred 
to a chain recurrent point as a pseudo-non-wandering point. The set of chain recurrent points for $\phi$ is well known to be a nonempty $\phi$-invariant closed subset of $X$.

The following characterization of the pointwise chain recurrence is an immediate consequence of $[10$, Lemma 2$]$ :

Lemma 3.3. For a homeomorphism $\phi$ on a compact Hausdorff space $X$, the following are equivalent:

(i) $\phi$ is pointwise chain recurrent;

(ii) There exist no open subset $U$ of $X$ such that $\phi(\bar{U})$ is a proper subset of $U$, where $\bar{U}$ denotes the closure of $U$.

The following proposition might remind us of an immediate consequence of [8, Theorem 5] that for a non-singular and bi-measurable transformation $T$ on a Lebesgue space $(\Omega, \mathcal{B}, \mu)$, the following notions in the measurable sense for $T$ are equivalent:

(i) $T$ is recurrent;

(ii) $T$ is incompressible;

(iii) There exist no set $U \in \mathcal{B}$ with $\mu(U)>0$ which is finitely Hopf-equivalent to a proper subset of $U$.

Proposition 3.4. For a homeomorphism $\phi$ on a zero-dimensional compact Hausdorff space $X$, the following are equivalent:

(i) $\phi$ is pointwise chain recurrent;

(ii) There exist no clopen subset $U$ of $X$ such that $\phi(U)$ is a proper subset of $U$;

(iii) There exist no clopen subset $U$ of $X$ which is finitely Hopf-equivalent to a proper clopen subset of $U$.

Proof. The equivalence of (ii) and (iii) is Lemma 2.6 itself. It follows from Lemma 3.3 that (i) implies (ii).

To prove that (ii) implies (i), it is enough for us to show that if the set of chain recurrent points for $\phi$ is a proper subset of $X$, then there exists a clopen subset $V$ of $X$ such that $\phi(V)$ is a proper subset of $V$. Suppose that the set of chain recurrent points for $\phi$ is a proper subset of $X$. By Lemma 3.3, there exists an open set $U \subset X$ such that $\phi(\bar{U})$ is a proper subset of $U$. There exists a collection $\left\{U_{\lambda} ; \lambda \in \Lambda\right\}$ of clopen subsets of $X$ such that $U=\bigcup_{\lambda \in \Lambda} U_{\lambda}$. Then, there exist finitely many indices $\left\{\lambda_{i} \in \Lambda ; 1 \leq i \leq k\right\}$ such that $\phi(\bar{U}) \subset \bigcup_{i=1}^{k} U_{\lambda_{i}}$. If $\phi(\bar{U})=\bigcup_{i=1}^{k} U_{\lambda_{i}}$, then we can take the set $\bar{U}$ to be the desired clopen set $V$. So, suppose that $\phi(\bar{U}) \subsetneq \bigcup_{i=1}^{k} U_{\lambda_{i}}$. Set $V=\bigcup_{i=1}^{k} U_{\lambda_{i}}$, which is clopen. Then we see that $\phi(V) \subset \phi(\bar{U}) \subsetneq V$. 


\section{Hitting times}

Let $\phi$ be a homeomorphism on a zero-dimensional compact Hausdorff space $X$. If any point in the union of two nonempty clopen subsets of $X$ sojourns in each of the sets equally often within finite time, one of the sets can be transformed by finite decomposition into the other along the orbits, which is established by the following key lemma of this paper:

Lemma 4.1. Let $A$ and $B$ be nonempty clopen subsets of $X$. If for every $x \in A$ there exists an integer $p \geq 1$ such that

$$
\sum_{j=0}^{p-1} \chi_{A}\left(\phi^{j} x\right) \leq \sum_{j=0}^{p-1} \chi_{B}\left(\phi^{j} x\right)
$$

then there exists an $\alpha \in[[\phi]]_{*}$ with $\operatorname{Dom}(\alpha)=A$ and $\operatorname{Im}(\alpha) \subset B$ whose time change function $t_{\alpha}: A \rightarrow \mathbb{Z}$ is given by

$$
t_{\alpha}(x)=\min \left\{p \geq 0: \sum_{j=0}^{p} \chi_{A}\left(\phi^{j} x\right)=\sum_{j=0}^{p} \chi_{B}\left(\phi^{j} x\right)\right\} \text { for } x \in A
$$

Proof. Fix an $x \in A$ arbitrarily for the moment. Define a function $f_{x}$ : $[1, \infty) \rightarrow \mathbb{R}$ so that

$$
f_{x}(n)=\sum_{j=0}^{n-1}\left(\chi_{B}\left(\phi^{j} x\right)-\chi_{A}\left(\phi^{j} x\right)\right) \text { for every integer } n \geq 1
$$

and that the graph of $f_{x}$ on each interval $[n, n+1], n \in \mathbb{N}$, is the line segment whose endpoints are $\left(n, f_{x}(n)\right)$ and $\left(n+1, f_{x}(n+1)\right)$. Notice that $f_{x}(1)=0$ if and only if $x \in A \cap B$, and $f_{x}(1)=-1$ if and only if $x \in A \backslash B$. The assumption of the lemma implies the existence of an integer $p \geq 1$ such that $f_{x}(p) \geq 0$. Hence, the continuity of $f_{x}$ implies the existence of an integer $1 \leq p^{\prime} \leq p$ such that $f_{x}\left(p^{\prime}\right)=0$. Hence, $n(x)=\min \left\{p \geq 1 ; f_{x}(p)=0\right\}$ is well-defined for any $x \in A$. Since a function

$$
\sum_{j=0}^{n-1} \chi_{A}\left(\phi^{j} \cdot\right)-\sum_{j=0}^{n-1} \chi_{B}\left(\phi^{j} \cdot\right)
$$

is an integer-valued continuous function for each integer $n \geq 1$, there exists a clopen neighborhood $U_{x} \subset A$ of each $x \in A$ such that $n(x)=n(y)$ for any $y \in U_{x}$. The compactness of $A$ shows that $A=\bigcup_{i=1}^{s} U_{x_{i}}$ for some finite set $\left\{x_{1}, \ldots, x_{s}\right\} \subset A$, which implies therefore that there exist a partition $\left\{A_{1}, \ldots, A_{k}\right\}$ of $A$ into clopen sets and integers $1 \leq p_{1}<\cdots<p_{k}$ such that $n(x)=p_{i}$ for any $x \in A_{i}$ and $1 \leq i \leq k$. 
Suppose that there exists an $x \in A$ such that $\phi^{n(x)-1} x \notin B$. This implies that $n(x)>1$. Then, it follows from the minimum property of $n(x)$ that $\phi^{n(x)-1} x \in A$. It follows therefore that $f_{x}(n(x))-f_{x}(n(x)-1)=-1$ and so $f_{x}(n(x)-1)=1$. The continuity of $f_{x}$ again implies the existence of an integer $1 \leq p<n(x)$ such that $f_{x}(p)=0$, that is,

$$
\sum_{j=0}^{p-1} \chi_{A}\left(\phi^{j} x\right)=\sum_{j=0}^{p-1} \chi_{B}\left(\phi^{j} x\right)
$$

which is a contradiction to the minimum property of $n(x)$.

Next, we shall assert that $\phi^{p_{1}-1} A_{1}, \phi^{p_{2}-1} A_{2}, \ldots, \phi^{p_{k}-1} A_{k}$ are pairwise disjoint. To the contrary, assume that there exist integers $1 \leq i<i^{\prime} \leq k$ such that $\phi^{p_{i}-1} A_{i} \cap \phi^{p_{i^{\prime}-1}} A_{i^{\prime}} \neq \emptyset$. It follows that there exists an $x \in A_{i^{\prime}}$ such that

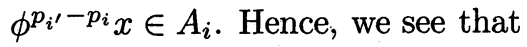

$$
\sum_{j=0}^{p_{i}-1}\left(\chi_{A}-\chi_{B}\right)\left(\phi^{j} \phi^{p_{i^{\prime}}-p_{i}} x\right)=0 \text { and } \sum_{j=0}^{p_{i^{\prime}}-1}\left(\chi_{A}-\chi_{B}\right)\left(\phi^{j} x\right)=0 .
$$

It follows therefore that

$$
\sum_{j=0}^{p_{i^{\prime}}-p_{i}-1}\left(\chi_{A}-\chi_{B}\right)\left(\phi^{j} x\right)=0
$$

which contradicts the minimum property of $p_{i^{\prime}}$. From the above arguments, we can define a $\phi$-homeomorphism $\alpha$ by

$$
\alpha(x)=\phi^{p_{i}-1}(x) \text { if } x \in A_{i}, \quad 1 \leq i \leq k,
$$

which clearly satisfies the desired properties.

\section{Relationships among the aforementioned conditions}

Throughout this section unless specified otherwise, we let $\phi$ be just a homeomorphism on a zero-dimensional compact Hausdorff space $X$.

Recall Conditions (a) (d) for a pair of nonempty clopen subsets $A$ and $B$ of $X:$

(a) $A$ and $B$ are finitely Hopf-equivalent;

(b) $A$ and $B$ are cohomologous, i.e., $\chi_{A}-\chi_{B}$ is a coboundary;

(c) $A$ and $B$ have positively equal hitting times, i.e., for any $x \in A \cup B$ there exists an integer $p \geq 1$ such that

$$
\sum_{j=0}^{p-1} \chi_{A}\left(\phi^{j} x\right)=\sum_{j=0}^{p-1} \chi_{B}\left(\phi^{j} x\right)
$$


(d) $A$ and $B$ are countably Hopf-equivalent.

In addition, we consider also Condition (e): for any $x \in A$ there exists an integer $p \geq 1$ such that

$$
\sum_{j=0}^{p-1} \chi_{A}\left(\phi^{j} x\right)=\sum_{j=0}^{p-1} \chi_{B}\left(\phi^{j} x\right),
$$

and for any $x \in B$ there exists an integer $q \geq 1$ such that

$$
\sum_{j=0}^{q-1} \chi_{A}\left(\phi^{-j} x\right)=\sum_{j=0}^{q-1} \chi_{B}\left(\phi^{-j} x\right)
$$

When this condition holds, we say that $A$ and $B$ have equal hitting times.

The following proposition gives one of the additional properties of $\phi$ which will make Condition (c) to be sufficient for Condition (a):

THEOREM 5.1. If $\phi$ is pointwise chain recurrent, then Condition (c) implies Condition (a).

Proof. By Lemma 4.1, there exist $\alpha, \beta \in[[\phi]]_{*}$ such that $\operatorname{Dom}(\alpha)=A$, $\operatorname{Im}(\alpha) \subset B, \operatorname{Dom}(\beta)=B$ and $\operatorname{Im}(\beta) \subset A$. The desired conclusion follows from Proposition 3.4.

Corollary 5.2. If $\phi$ is pointwise positively recurrent, then Condition (c) implies Condition (a).

Proof. Any positively recurrent point for $\phi$ is chain recurrent for $\phi$.

If we assume no such additional properties of $\phi$ as in Theorem 5.1, then we cannot expect more than the following:

Theorem 5.3. Condition (c) implies Condition (d).

Proof. By Lemma 4.1, we obtain $\phi$-homeomorphisms $\alpha, \beta \in[[\phi]]_{*}$ such that $\operatorname{Dom}(\alpha)=A, \operatorname{Im}(\alpha) \subset B, \operatorname{Dom}(\beta)=B$ and $\operatorname{Im}(\beta) \subset A$. It follows from the proof of Lemma 4.1 that both of the continuous time change functions $t_{\alpha}$ and $t_{\beta}$ of $\alpha$ and $\beta$ are non-negative. The Schroeder-Bernstein type argument shows that $A$ is decomposed into countable pairwise disjoint closed sets $E_{i}, i \geq 0, F_{i}, i \geq 0$ and $G$ which are defined by

$$
\begin{aligned}
& E_{i}=(\beta \alpha)^{i}(A) \backslash(\beta \alpha)^{i} \beta(B), i \geq 0 \\
& F_{i}=(\beta \alpha)^{i} \beta(B) \backslash(\beta \alpha)^{i+1}(A), i \geq 0 \\
& G=\bigcap_{i=0}^{\infty}(\beta \alpha)^{i}(A),
\end{aligned}
$$


and that $B$ is decomposed into countable pairwise disjoint closed sets $\alpha\left(E_{i}\right), i \geq 0$, $\beta^{-1}\left(F_{i}\right), i \geq 0$ and $\alpha(G)$. Since the sets $t_{\alpha}(A)$ and $t_{\beta}(B)$ are finite, we can write

$$
t_{\alpha}(A)=\left\{0 \leq t_{1}<t_{2}<\cdots<t_{k}\right\}, t_{\beta}(B)=\left\{0 \leq s_{1}<s_{2}<\cdots<s_{l}\right\}
$$

and then we define

$$
A_{j}=t_{\alpha}^{-1}\left\{t_{j}\right\} \text { for } 1 \leq j \leq k, \text { and } B_{m}=t_{\beta}^{-1}\left\{s_{m}\right\} \text { for } 1 \leq m \leq l
$$

We see that $A$ is decomposed into countably many pairwise disjoint closed sets

$$
\left\{E_{i} \cap A_{j}, G \cap A_{j}, F_{i} \cap \beta\left(B_{m}\right) ; i \geq 0,1 \leq j \leq k, 1 \leq m \leq l\right\}
$$

and $B$ is decomposed into countably many pairwise disjoint closed sets

$$
\left\{\phi^{t_{j}}\left(E_{i} \cap A_{j}\right), \phi^{t_{j}}\left(G \cap A_{j}\right), \phi^{-s_{m}}\left(F_{i} \cap \beta\left(B_{m}\right)\right) ; i \geq 0,1 \leq j \leq k, 1 \leq m \leq l\right\} .
$$

REMARK 5.4. In view of the above proof, we have a (measurable) bijection $\xi: A \rightarrow B$ such that

$$
\xi(x)= \begin{cases}\phi^{t_{\alpha}(x)}(x) & \text { if } x \in \bigcup_{i=1}^{\infty} E_{i} \cup G \\ \phi^{-t_{\beta}\left(\beta^{-1}(x)\right)}(x) & \text { if } x \in \bigcup_{i=1}^{\infty} F_{i}\end{cases}
$$

We cannot conclude $\xi$ to be continuous because both $t_{\alpha}$ and $t_{\beta}$ are non-negative. This is the reason why Condition (c) does not necessarily imply Condition (a) without some additional properties of $\phi$.

The following says that Condition (e) which is similar to (c) is sufficient for (a) under no additional properties of $\phi$ :

Proposition 5.5. Condition (e) implies Condition (a).

Proof. By Lemma 4.1, there exists an $\alpha \in[[\phi]]_{*}$ with $\operatorname{Dom}(\alpha)=A$ and $\operatorname{Im}(\alpha) \subset B$ whose time change function $t_{\alpha}: A \rightarrow \mathbb{Z}_{+}$is given by

$$
t_{\alpha}(x)=\min \left\{p \geq 0 ; \sum_{j=0}^{p} \chi_{A}\left(\phi^{j} x\right)=\sum_{j=0}^{p} \chi_{B}\left(\phi^{j} x\right)\right\} \quad \text { for } x \in A
$$


By using an argument similar to that in the proof of Lemma 4.1, we see that there exists a $\beta \in[[\phi]]_{*}$ with $\operatorname{Dom}(\beta)=B$ and $\operatorname{Im}(\beta) \subset A$ whose time change function $t_{\beta}: B \rightarrow(-\infty, 0]$ is given by

$$
t_{\beta}(x)=-\min \left\{p \geq 0 ; \sum_{j=0}^{p} \chi_{A}\left(\phi^{-j} x\right)=\sum_{j=0}^{p} \chi_{B}\left(\phi^{-j} x\right)\right\} \quad \text { for } x \in B
$$

For these $\alpha$ and $\beta$, choose $E_{i}, F_{i}, i \geq 0$, and $G$ as in the proof of Theorem 5.3. Define a bijective mapping $\gamma: A \rightarrow B$ by

$$
\gamma(x)= \begin{cases}\alpha(x) & \text { if } x \in G \cup \bigcup_{i=0}^{\infty} E_{i} \\ \beta^{-1}(x) & \text { if } x \in \bigcup_{i=0}^{\infty} F_{i} .\end{cases}
$$

If we define $t_{\gamma}: A \rightarrow \mathbb{Z}$ by

$$
t_{\gamma}(x)= \begin{cases}t_{\alpha}(x) & \text { if } x \in G \cup \bigcup_{i=0}^{\infty} E_{i} \\ -t_{\beta}\left(\beta^{-1}(x)\right) & \text { if } x \in \bigcup_{i=0}^{\infty} F_{i}\end{cases}
$$

then we obtain that $\gamma(x)=\phi^{t_{\gamma}(x)}(x)$ for any $x \in A$. Since each of $E_{i}$ and $F_{i}$, $i \geq 0$, is clopen, the discontinuity points of $t_{\gamma}$ must lie in the boundary of $G$. If $x_{0}$ is a discontinuity point of $t_{\gamma}$, then there exists a sequence $\left\{x_{n}\right\}_{n \geq 1} \subset \bigcup_{i=0}^{\infty} F_{i}$ with $x_{n} \rightarrow x_{0}$ as $n \rightarrow \infty$ such that $t_{\gamma}\left(x_{0}\right) \neq t_{\gamma}\left(x_{n}\right)$ for every $n \geq 1$, i.e., $t_{\alpha}\left(x_{0}\right) \neq$ $-t_{\beta}\left(\beta^{-1}\left(x_{n}\right)\right)$ for every $n \geq 1$. However, this cannot happen because both $t_{\alpha}$ and $t_{\beta}$ are continuous and it follows from (1) and (2) that $t_{\alpha}\left(x_{0}\right)=-t_{\beta}\left(\beta^{-1}\left(x_{0}\right)\right)$. Thus, $t_{\gamma}$ has no discontinuity points.

In the rest of this paper, we will see some connections among recurrence properties of $\phi$ and various conditions on a pair of nonempty clopen subsets $A$ and $B$ of $X$ :

(a) $A$ and $B$ are finitely Hopf-equivalent;

(b) $A$ and $B$ are cohomologous;

(c) $A$ and $B$ have positively equal hitting times;

(d) $A$ and $B$ are countably Hopf-equivalent;

(e) $A$ and $B$ have equal hitting times.

THEOREM 5.6. (i) $\phi$ is pointwise positively recurrent if and only if Condition (b) implies Condition (c); 
(ii) $\phi$ is pointwise recurrent, hence pointwise uniformly recurrent, if and only if Condition (b) implies Condition (e).

Proof. (i): Suppose that $\phi$ is pointwise positively recurrent. We shall show that Condition (b) implies Condition (c). Suppose that nonempty clopen subsets $A$ and $B$ of $X$ satisfies that $\chi_{A}-\chi_{B}=f \circ \phi-f$ for some integer-valued continuous function $f$ on $X$, and fix an arbitrary point $x_{0} \in A \cup B$. Take a clopen neighborhood $U$ of $x_{0}$ on which values of $f$ are constant. There exists an integer $p_{0} \geq 1$ with $\phi^{p_{0}} x \in U$. Then,

$$
\sum_{j=0}^{p_{0}-1} \chi_{A}\left(\phi^{j} x_{0}\right)-\sum_{j=0}^{p_{0}-1} \chi_{B}\left(\phi^{j} x_{0}\right)=f\left(\phi^{p_{0}} x_{0}\right)-f\left(x_{0}\right)=0 .
$$

To show the converse, suppose that Condition (b) implies Condition (c). Let $B \subset X$ be an arbitrary nonempty clopen set and fix an $x_{0} \in B$ arbitrarily, and furthermore put $A=\phi^{-1} B$. Since $\chi_{A}-\chi_{B}=\chi_{B} \circ \phi-\chi_{B}$, there exists an integer $p \geq 1$ such that

$$
\sum_{j=0}^{p-1} \chi_{A}\left(\phi^{j} x_{0}\right)=\sum_{j=0}^{p-1} \chi_{B}\left(\phi^{j} x_{0}\right)
$$

We obtain that $\chi_{B}\left(\phi^{p} x_{0}\right)-\chi_{B}\left(x_{0}\right)=\sum_{j=0}^{p-1} \chi_{A}\left(\phi^{j} x_{0}\right)-\sum_{j=0}^{p-1} \chi_{B}\left(\phi^{j} x_{0}\right)=0$ and thus $\phi^{p} x_{0} \in B$.

(ii): Almost the same argument as in the proof of (i) will work.

Corollary 5.7. (i) $\phi$ is pointwise positively recurrent if and only if Conditions (a), (b) and (c) are all equivalent;

(ii) $\phi$ is pointwise recurrent, hence pointwise uniformly recurrent, if and only if Conditions (a), (b), (c) and (e) are all equivalent.

Proof. It is always true that Condition (a) implies Condition (b).

(i): Suppose that $\phi$ is pointwise positively recurrent. It follows from (i) of the above theorem that Condition (b) implies Condition (c). It follows from Corollary 5.2 that Condition (c) implies Condition (a). Conversely, again by (i) of the above theorem, the equivalence of Conditions (a), (b) and (c) implies the pointwise positive recurrence of $\phi$.

(ii): Suppose that $\phi$ is pointwise recurrent. Then, Conditions (a), (b) and (c) are all equivalent from (i) of this corollary. The equivalence of Conditions (b) and (e) is obtained by (ii) of the above theorem.

REMARK 5.8. If $\phi$ is a minimal homeomorphism on a Cantor set $X$, then we see from the above corollary that Conditions (a) and (c) are equivalent. For such a homeomorphism, S. Bezuglyi and J. Kwiatkowski gave in [1, Theorem 2.6] another condition equivalent to Condition (a) which restricts the positive equal 
hitting time $n$ implementing Condition (c) to those appearing in a "cycle" of a Kakutani-Rokhlin partition.

There exists a class of homeomorphisms on zero-dimensional compact Hausdorff spaces for which (a), (b) and (c) are not equivalent but (b) implies (a). Let $\phi$ be an almost minimal homeomorphism in the sense of [2] on a Cantor set $X$ and denote by $*$ the unique fixed point of $\phi$. Since for any nonempty clopen set $A \subset X \backslash\{*\}$, the set $\left\{x \in A ; \phi^{n} x \notin A\right.$ for every $\left.n \geq 1\right\}$ is nonempty [2, Proposition 2.2], (a), (b) and (c) can never be equivalent for $\phi$. On the other hand, we can show in the same way as in [4, Lemma 3.3] that (b) implies (a). Let $A, B \subset X$ be nonempty disjoint clopen sets such that $\chi_{A}-\chi_{B}=f \circ \phi-f$ for some integer-valued continuous function $f$ on $X$. Since $(X, \phi, *)$ is essentially minimal in the sense of [7, Definition 1.2], [7, Lemma 4.1] implies the existence of a Kakutani-Rokhlin partition $\mathcal{P}=\{Z(k, j) ; 1 \leq j \leq J(k), 1 \leq k \leq K\}$ such that $\mathcal{P}$ is finer than $\{X \backslash(A \cup B), A, B\}$ as a partition; $f$ is constant on $\bigcup_{k} Z(k, 1) ; * \in \bigcup_{k} Z(k, J(k))$. Then, we see that for every $1 \leq k \leq K$

$$
\sharp\{1 \leq j \leq J(K) ; Z(k, j) \subset A\}=\sharp\{1 \leq j \leq J(K) ; Z(k, j) \subset B\},
$$

which implies that $A$ and $B$ are finitely Hopf-equivalent.

\section{References}

[1] S. Bezuglyi and J. Kwiatkowski, The topological full group of a Cantor minimal system is dense in the full group, Topol. Methods Nonlinear Anal., 16-2 (2000), 371-397.

[2] A. Danilenko, Strong orbit equivalence of locally compact Cantor minimal systems, Internat. J. Math., 12-1 (2001), 113-123.

[3] T. Giordano, I. Putnam and C. Skau, Topological orbit equivalence and $C^{*}$-crossed products, J. Reine Angew. Math., 469 (1995), 51-111.

[4] T. Giordano, I.F. Putnam and C.F. Skau, Full groups of Cantor minimal systems, Israel J. Math., 111 (1999), 285-320.

[5] E. Glasner and B. Weiss, Weak orbit equivalence of Cantor minimal systems, Internat. J. Math., 6 (1995), 559-579.

[6] T. Hamachi and M. Osikawa, Ergodic groups of automorphisms and Krieger's theorems, Seminar on Mathematical Sciences, Vol. 3, Keio University, Department of Mathematics, Yokohama, 1981.

[ 7 ] R.H. Herman, I.F. Putnam and C.F. Skau, Ordered Bratteli diagrams, dimension groups and topological dynamics, Internat. J. Math., 3 (1992), 827-864.

[ 8] E. Hopf, Theory of measure and invariant integrals, Trans. Amer. Math. Soc., 34-2 (1932), 373-393.

[9] K. Nagami, Dimension theory, Pure and Applied Mathematics, Vol. 37, Academic Press, New York and London, 1970, with an appendix by Y. Kodama.

[10] M.V. Pimsner, Embedding some transformation group $C^{*}$-algebras into AF-algebras, Ergodic Theory Dynam. Systems, 3-4 (1983), 613-626. 
Department of Mathematics Faculty of Science and Technology KEIO UNIVERSITY

3-14-1, HiYoshI, КOHOKU-KU YOKOHAMA, JAPAN

E-mail: hisatoc@math.keio.ac.jp 